\title{
Boundary Value Problem of Nonlinear Hybrid Differential Equations with Linear and Nonlinear Perturbations
}

\author{
Said Melliani, Abdelati El Allaoui $\mathbb{D}$, and Lalla Saadia Chadli \\ Sultan Moulay Slimane University, Department of Mathematics, Laboratory of Applied Mathematics \& Scientific Calculus, \\ P.O. Box 523, Beni Mellal, Morocco \\ Correspondence should be addressed to Abdelati El Allaoui; elallaoui199@gmail.com
}

Received 12 July 2020; Revised 25 August 2020; Accepted 4 September 2020; Published 22 September 2020

Academic Editor: Patricia J. Y. Wong

Copyright ( 2020 Said Melliani et al. This is an open access article distributed under the Creative Commons Attribution License, which permits unrestricted use, distribution, and reproduction in any medium, provided the original work is properly cited.

The aim of this paper is to study a boundary value problem of the hybrid differential equation with linear and nonlinear perturbations. It generalizes the existing problem of second type. The existence result is constructed using the Leray-Schauder alternative, and the uniqueness is guaranteed by Banach's fixed-point theorem. Towards the end of this paper, an example is provided to illustrate the obtained results.

\section{Introduction}

The application of differential equations in different real domains has increased the importance of this theory which is still under development. Hybrid differential equations are a subfield of differential equations which also has enough importance. Recently, it has attracted the attention of several mathematicians [1-5]. In [6], the authors studied the following hybrid differential equation with linear perturbation:

$$
\left\{\begin{array}{l}
\frac{\mathrm{d}}{\mathrm{d} t}[x(t)-f(t, x(t))]=g(t, x(t)) \\
x(0)=x_{0}
\end{array}\right.
$$

where the existence of solutions to these problems has been ensured using Dhage's theorem.

Motivated by the abovementioned problem, we consider the following boundary value problem for hybrid differential equation:

$$
\left\{\begin{array}{l}
\frac{\mathrm{d}}{\mathrm{d} t}[x(t) f(t, x(t))-g(t, x(t))]=h(t, x(t)), \quad t \in I=[0, a], a>0, \\
x(0) f(0, x(0))+\alpha x(a) f(a, x(a))=g(0, x(0))+\alpha g(a, x(a))+\beta,
\end{array}\right.
$$

where $f \in \mathscr{C}(I \times \mathbb{R} ; \mathbb{R} /\{0\})$ and $g, h \in \mathscr{C}(I \times \mathbb{R} ; \mathbb{R})$ are given functions and $\alpha, \beta \in \mathbb{R}$ such that $\alpha \neq-1$.

The proposed problem can be considered as generalization of problem (1) which becomes a special case if we take $f=1$, and also, the novelty is at the level of the relationship between the boundary values. Using Banach's fixed- point theorem, we show the existence and the uniqueness of the solution of the proposed problem.

The fixed-point theorems used for hybrid differential equations with perturbation of first or second type are those based on the composition of the solution as sum or product of two operators such as the Dhage case. For our case, we 
have a mixed problem which brings together the two types, where we thought of using Leray-Schauder's Fixed-Point Theorem as a second existence result for which we will have a single operator.

\section{Preliminaries}

First, we recall some basic results used in this paper. We start by recalling Leray-Schauder alternative.

Lemma 1 (see [7]).

Let $\Pi: Y \longrightarrow Y$ be a completely continuous operator and

$$
\mathscr{P}_{\Pi}=\{y \in Y: y=\delta \Pi y \text { for some } 0<\delta<1\} .
$$

Then, either the set $\mathscr{P}_{\Pi}$ is unbounded or $\Pi$ has at least one fixed point.

Now, we recall the following lemmas on which we will base ourselves to build the solution of our problem.

Lemma 2 (see [6]). Suppose that $x \mapsto x-g(t, x)$ is increasing in $\mathbb{R}$ for each $t \in I$. Then, for any $h: I \longrightarrow \mathbb{R}_{+}$, the function $x \in \mathscr{C}\left(J, \mathbb{R}_{+}\right)$is a solution of the hybrid differential equation

$$
\left\{\begin{array}{l}
\frac{\mathrm{d}}{\mathrm{d} t}[x(t)-g(t, x(t))]=h(t), \quad t \in I, \\
x(0)=x_{0} \in \mathbb{R},
\end{array}\right.
$$

if and only if $x$ satisfies the following hybrid integral equation:

$$
x(t)=x_{0}-g\left(0, x_{0}\right)+g(t, x(t))+\int_{0}^{t}(h, s) \mathrm{d} s, \quad t \in J .
$$

\section{Existence Result}

Before presenting the existence results, we pose the following hypotheses:

(i) The map $x \longmapsto x f(t, x)-g(t, x)$ is increasing in $\mathbb{R}$ for each $t \in I$.

(ii) There exist positive constants $v_{f}$ and $\mu_{g}$, such that

$$
\begin{aligned}
& |f(t, x)| \geq v_{f}, \\
& |g(t, x)| \leq \mu_{g} .
\end{aligned}
$$

(iii) There exists positive constants $\lambda_{f}, \lambda_{g}$, and $\lambda_{h}$ such that

$$
\begin{aligned}
& |f(t, x)-f(t, y)| \leq \lambda_{f}|x-y|, \\
& |g(t, x)-g(t, y)| \leq \lambda_{g}|x-y|, \\
& |h(t, x)-h(t, y)| \leq \lambda_{h}|x-y|,
\end{aligned}
$$

for each $t \in I$ and $x, y \in \mathbb{R}$.
Denote $\mathscr{C}:=\mathscr{C}(I, \mathbb{R})$, the space of all continuous mapping defined on $I$ into $\mathbb{R}$ endowed with the norm $\|x\|=\sup _{t \in I}\|x(t)\|$.

Lemma 3. Let $h \in \mathscr{C}(I, \mathbb{R})$, then $x$ is an integral solution of (2) if and only if it satisfies the following integral equation:

$$
\begin{aligned}
x(t)= & \frac{\beta}{(1+\alpha) f(t, x(t))}-\frac{\alpha}{(1+\alpha) f(t, x(t))} \\
& \cdot \int_{0}^{a}(h, s, x(s)) \mathrm{d} s+\frac{g(t, x(t))}{f(t, x(t))}+\frac{1}{f(t, x(t))} \\
& \cdot \int_{0}^{t}(h, s, x(s)) \mathrm{d} s,
\end{aligned}
$$

for each $t \in I$.

Proof. Suppose that $x$ is a solution for (2), then we obtain

$$
\begin{aligned}
x(t) f(t, x(t))= & x(0) f(0, x(0))-g(0, x(0))+g(t, x(t)) \\
& +\int_{0}^{t} h(s, x(s)) \mathrm{d} s, \quad \text { for } t \in I .
\end{aligned}
$$

Then,

$$
\begin{aligned}
\alpha x(a) f(a, x(a))= & \alpha x(0) f(0, x(0))-\alpha g(0, x(0)) \\
& +\alpha g(a, x(a))+\alpha \int_{0}^{a} h(s, x(s)) \mathrm{d} s .
\end{aligned}
$$

Hence,

$$
\begin{aligned}
x(0) f(0, x(0))+\alpha x(a) f(a, x(a))= & (1+\alpha) x(0) f(0, x(0)) \\
& -\alpha g(0, x(0)) \\
& +\alpha g(a, x(a)) \\
& +\alpha \int_{0}^{a} h(s, x(s)) \mathrm{d} s .
\end{aligned}
$$

By using the second equation in (2), we obtain

$$
x(0) f(0, x(0))-g(0, x(0))=\frac{\beta}{1+\alpha}-\frac{\alpha}{1+\alpha} \int_{0}^{a} h(s, x(s)) \mathrm{d} s .
$$

By replacing in (9), we obtain

$$
\begin{aligned}
x(t)= & \frac{\beta}{(1+\alpha) f(t, x(t))}-\frac{\alpha}{(1+\alpha) f(t, x(t))} \int_{0}^{a} h(s, x(s)) \mathrm{d} s \\
& +\frac{g(t, x(t))}{f(t, x(t))}+\frac{1}{f(t, x(t))} \int_{0}^{t} h(s, x(s)) \mathrm{d} s, \quad t \in I .
\end{aligned}
$$

The other implication is trivial.

Now, we can give the definition of an integral solution of problem (2). 
Definition 1. An integral solution of problem (2) is a $\quad$ (2) $x$ satisfies the following integral equation: function $x \in \mathscr{C}$ which satisfies the following:

(1) The map $t \longmapsto x f(t, x)-g(t, x)$ is continuous for each $x \in \mathbb{R}$, and

$$
\begin{aligned}
x(t)= & \frac{\beta}{(1+\alpha) f(t, x(t))}-\frac{\alpha}{(1+\alpha) f(t, x(t))} \int_{0}^{a} h(s, x(s)) \mathrm{d} s+\frac{g(t, x(t))}{f(t, x(t))} \\
& +\frac{1}{f(t, x(t))} \int_{0}^{t} h(s, x(s)) \mathrm{d} s,
\end{aligned}
$$

for each $t \in I$.

To reduce the form of mathematical expressions, consider the following notations:

$$
\begin{aligned}
& \pi_{1}=|\beta|+a k_{h}|\alpha|+\left(\mu_{g}+a k_{h}\right)|1+\alpha|, \\
& \pi_{2}=v_{f}|1+\alpha|, \\
& \pi_{3}=a \lambda_{h}(|\alpha|+|1+\alpha|), \\
& \omega=\frac{\lambda_{f}|\beta|+\left(v_{f} \lambda_{g}+\lambda_{f} \mu_{g}\right)|1+\alpha|+a\left(v_{f} \lambda_{h}+\lambda_{f}\left(\lambda_{h} r+k_{h}\right)\right)(|\alpha|+|1+\alpha|)}{v_{f}^{2}|1+\alpha|},
\end{aligned}
$$

where $r$ is a real number which will be defined later.

Now, we can provide our first existence result.

Theorem 1. Suppose that $\left(A_{0}\right)-\left(A_{3}\right)$ are satisfied. In addition, assume that the following condition is verified:

$$
\max \left\{\omega, \frac{\pi_{3}}{\pi_{2}}\right\}<1
$$

Proof. First, we define the following closed ball:

$$
B_{r}=\{x \in \mathscr{P} \mathscr{C}:\|x\| \leq r\},
$$

where

$$
r \geq \frac{\pi_{1}}{\pi_{2}-\pi_{3}} .
$$

Also, we define the following operator $\Pi$ on $\mathscr{C}$ by

Then, the problem (2) has a unique solution.

$$
\Pi x(t)=\frac{\beta}{(1+\alpha) f(t, x(t))}-\frac{\alpha}{(1+\alpha) f(t, x(t))} \int_{0}^{a} h(s, x(s)) \mathrm{d} s+\frac{g(t, x(t))}{f(t, x(t))}+\frac{1}{f(t, x(t))} \int_{0}^{t} h(s, x(s)) \mathrm{d} s,
$$

for each $t \in I$.

The proof will be made in two steps: 
(i) $\Pi B_{r} \subseteq B_{r}$. Indeed, for $x \in B_{r}$ and $t \in I$, we have

$$
\begin{aligned}
|\Pi(x)(t)| & \leq \frac{|\beta|}{|1+\alpha||f(t, x(t))|}+\frac{|\alpha|}{|1+\alpha||f(t, x(t))|} \int_{0}^{a}(|h(s, x(s))-h(s, 0)|+|h(s, 0)|) \mathrm{d} s \\
& +\frac{|g(t, x(t))|}{|f(t, x(t))|}+\frac{1}{|f(t, x(t))|} \int_{0}^{t}(|h(s, x(s))-h(s, 0)|+|h(s, 0)|) \mathrm{d} s \\
& \leq \frac{|\beta|}{v_{f}|1+\alpha|}+\frac{|\alpha|}{v_{f}|1+\alpha|} \int_{0}^{a}\left(\lambda_{h}|x(s)|+k_{h}\right) \mathrm{d} s \\
& +\frac{\mu_{g}}{v_{f}}+\frac{1}{v_{f}} \int_{0}^{t}\left(\lambda_{h}|x(s)|+k_{h}\right) \mathrm{d} s \\
& \leq \frac{|\beta|+a k_{h}|\alpha|}{v_{f}|1+\alpha|}+\frac{\mu_{g}+a k_{h}}{v_{f}}+\frac{a \lambda_{h}(|\alpha|+|1+\alpha|)}{v_{f}|1+\alpha|} r \\
& \leq \frac{\pi_{1}}{\pi_{2}}+\frac{\pi_{3}}{\pi_{2}} r .
\end{aligned}
$$

(ii) Hence, according to (18), we obtain

$$
\|\Pi(x)\| \leq r .
$$

$$
|\Pi(x)(t)| \leq\left(1-\frac{\pi_{3}}{\pi_{2}}\right) r+\frac{\pi_{3}}{\pi_{2}} r \leq r .
$$

(iv) $\Pi$ is a contraction:

For $x, y \in B_{r}$ and $t \in I$, we have

(iii) Then, 


$$
\begin{aligned}
& |\Pi(x)(t)-\Pi(y)(t)| \leq\left|\frac{\beta}{(1+\alpha) f(t, x(t))}-\frac{\beta}{(1+\alpha) f(t, y(t))}\right| \\
& +\left|\frac{\alpha}{(1+\alpha) f(t, x(t))} \int_{0}^{a} h(s, x(s)) \mathrm{d} s-\frac{\alpha}{(1+\alpha) f(t, x(t))} \int_{0}^{a} h(s, y(s)) \mathrm{d} s\right| \\
& +\left|\frac{\alpha}{(1+\alpha) f(t, x(t))} \int_{0}^{a} h(s, y(s)) \mathrm{d} s-\frac{\alpha}{(1+\alpha) f(t, y(t))} \int_{0}^{a} h(s, y(s)) \mathrm{d} s\right| \\
& +\left|\frac{g(t, x(t))}{f(t, x(t))}-\frac{g(t, y(t))}{f(t, x(t))}\right|+\left|\frac{g(t, y(t))}{f(t, x(t))}-\frac{g(t, y(t))}{f(t, y(t))}\right| \\
& +\left|\frac{1}{f(t, x(t))} \int_{0}^{t} h(s, x(s)) \mathrm{d} s-\frac{1}{f(t, x(t))} \int_{0}^{t} h(s, y(s)) \mathrm{d} s\right| \\
& +\left|\frac{1}{f(t, x(t))} \int_{0}^{t} h(s, y(s)) \mathrm{d} s-\frac{1}{f(t, y(t))} \int_{0}^{t} h(s, y(s)) \mathrm{d} s\right| \\
& \leq \frac{|\beta|}{v_{f}^{2}|1+\alpha|}|f(t, x(t))-f(t, y(t))|+\frac{|\alpha|}{v_{f}|1+\alpha|} \int_{0}^{a}|h(s, x(s))-h(s, y(s))| \mathrm{d} s \\
& +\frac{|\alpha|}{v_{f}^{2}|1+\alpha|} \int_{0}^{a}(|h(s, y(s))-h(s, 0)|+|h(s, 0)|) \mathrm{d} s|f(t, x(t))-f(t, y(t))| \\
& +\frac{1}{v_{f}}|g(t, x(t))-g(t, y(t))|+\frac{\mu_{g}}{v_{f}^{2}}|f(t, x(t))-f(t, y(t))| \\
& +\frac{1}{v_{f}} \int_{0}^{t}|h(s, x(s))-h(s, y(s))| \mathrm{d} s \\
& +\frac{1}{v_{f}^{2}} \int_{0}^{t}(|h(s, y(s))-h(s, 0)|+|h(s, 0)|) \mathrm{d} s|f(t, x(t))-f(t, y(t))| \\
& \leq\left(\frac{\lambda_{f}|\beta|}{v_{f}^{2}|1+\alpha|}+\frac{a \lambda_{h}|\alpha|}{v_{f}|1+\alpha|}+\frac{a \lambda_{f}|\alpha|\left(\lambda_{h} r+k_{h}\right)}{v_{f}^{2}|1+\alpha|}+\frac{\lambda_{g}}{v_{f}}+\frac{\lambda_{f} \mu_{g}}{v_{f}^{2}}+\frac{a \lambda_{h}}{v_{f}}+\frac{a \lambda_{f}\left(\lambda_{h} r+k_{h}\right)}{v_{f}^{2}}\right)\|x-y\| \leq \omega\|x-y\| .
\end{aligned}
$$

which shows $\Pi$ is a contraction.

Thus, $\Pi$ is a contraction. Then, the existence and uniqueness of the solution is guaranteed by Banach's fixedpoint theorem.

Now, we present the second existence result using Leray-Schauder alternative.

Theorem 2. Suppose that $\left(A_{0}\right)$ and $\left(A_{1}\right)$ are satisfied. In addition, assume that there exist $\gamma_{1}, \gamma_{2}>0$, such that

$$
|h(t, x)| \leq \gamma_{1}+\gamma_{2}\|x\| \text {, for each }(t, x) \in I \times \mathbb{R} .
$$

Also, $v_{f}>a \gamma_{2}$. Then, problem (2) has at least one solution.

Proof. Let $\mathscr{A} \subseteq \mathscr{C}$ be a bounded subset.

Then, there exists $\eta_{h}>0$ such that

$$
|h(t, x(t))| \leq \eta_{h} .
$$

for each $x \in \mathscr{A}$ and $t \in I$.

The proof will be given in several steps:

(i) $\Pi$ is uniformly bounded:

For $x \in \mathscr{A}$ and $t \in I$, we have

$$
\begin{aligned}
|\Pi(x)(t)| \leq & \frac{|\beta|}{|1+\alpha||f(t, x(t))|}+\frac{|\alpha|}{|1+\alpha||f(t, x(t))|} \\
& \cdot \int_{0}^{a}|h(s, x(s))| \mathrm{d} s+\frac{|g(t, x(t))|}{|f(t, x(t))|} \\
& +\frac{1}{|f(t, x(t))|} \int_{0}^{t}|h(s, x(s))| \mathrm{d} s \leq \frac{|\beta|+a \eta_{h}|\alpha|}{v_{f}|1+\alpha|} \\
& +\frac{\mu_{g}+a \eta_{h}}{v_{f}} .
\end{aligned}
$$

Then, $\Pi$ is uniformly bounded. 
(ii) $\Pi$ is equicontinuous:

$$
\begin{aligned}
& \left|\Pi(x)\left(\tau_{2}\right)-\Pi(x)\left(\tau_{1}\right)\right| \leq\left|\frac{\beta}{(1+\alpha) f\left(\tau_{1}, x\left(\tau_{1}\right)\right)}-\frac{\beta}{(1+\alpha) f\left(\tau_{2}, x\left(\tau_{2}\right)\right)}\right| \\
& +\left|\frac{\alpha}{(1+\alpha) f\left(\tau_{1}, x\left(\tau_{1}\right)\right)}-\frac{\alpha}{(1+\alpha) f\left(\tau_{2}, x\left(\tau_{2}\right)\right)}\right| \int_{0}^{a}|h(s, x(s))| \mathrm{d} s \\
& +\left|\frac{g\left(\tau_{1}, x\left(\tau_{1}\right)\right)}{f\left(\tau_{1}, x\left(\tau_{1}\right)\right)}-\frac{g\left(\tau_{2}, x\left(\tau_{2}\right)\right)}{f\left(\tau_{1}, x\left(\tau_{1}\right)\right)}\right|+\left|\frac{g\left(\tau_{2}, x\left(\tau_{2}\right)\right)}{f\left(\tau_{1}, x\left(\tau_{1}\right)\right)}-\frac{g\left(\tau_{2}, x\left(\tau_{2}\right)\right)}{f\left(\tau_{2}, x\left(\tau_{2}\right)\right)}\right| \\
& +\left|\frac{1}{f\left(\tau_{1}, x\left(\tau_{1}\right)\right)} \int_{0}^{\tau_{1}} h(s, x(s)) \mathrm{d} s-\frac{1}{f\left(\tau_{1}, x\left(\tau_{1}\right)\right)} \int_{0}^{\tau_{2}} h(s, x(s)) \mathrm{d} s\right| \\
& +\left|\frac{1}{f\left(\tau_{1}, x\left(\tau_{1}\right)\right)} \int_{0}^{\tau_{2}} h(s, x(s)) \mathrm{d} s-\frac{1}{f\left(\tau_{2}, x\left(\tau_{2}\right)\right)} \int_{0}^{\tau_{2}} h(s, x(s)) \mathrm{d} s\right| \\
& \leq \frac{|\beta|+a \eta_{h}|\alpha|}{v_{f}^{2}|1+\alpha|}\left|f\left(\tau_{1}, x\left(\tau_{1}\right)\right)-f\left(\tau_{2}, x\left(\tau_{2}\right)\right)\right|+\frac{1}{v_{f}}\left|g\left(\tau_{1}, x\left(\tau_{1}\right)\right)-g\left(\tau_{2}, x\left(\tau_{2}\right)\right)\right| \\
& +\frac{\mu_{g}}{\nu_{f}^{2}}\left|f\left(\tau_{1}, x\left(\tau_{1}\right)\right)-f\left(\tau_{2}, x\left(\tau_{2}\right)\right)\right|+\frac{\eta_{h}}{\nu_{f}}\left(\tau_{2}-\tau_{1}\right)+\frac{\tau_{2} \eta_{h}}{\nu_{f}^{2}}\left|f\left(\tau_{1}, x\left(\tau_{1}\right)\right)-f\left(\tau_{2}, x\left(\tau_{2}\right)\right)\right| \longrightarrow 0, \\
& \text { as } t_{2} \longrightarrow t_{1}
\end{aligned}
$$

Hence, $\Pi$ is equicontinuous.

(iii) $\mathscr{P}_{\Pi}$ is bounded:

We denote by

$$
\mathscr{P}_{\Pi}=\{x \in \mathbb{R}: x=\delta \Pi(x), 0 \leq \delta \leq 1\} .
$$

Let $x \in \mathscr{P}_{\Pi}$ and $t \in I$, we have

$$
|x(t)| \leq \frac{|\beta|+a \eta_{h}|\alpha|}{\nu_{f}|1+\alpha|}+\frac{\mu_{g}}{v_{f}}+\frac{a}{v_{f}}\left(\gamma_{1}+\gamma_{2}\|x\|\right),
$$

which implies that

$$
\|x\| \leq \frac{|\beta|+a \eta_{h}|\alpha|}{v_{f}|1+\alpha|}+\frac{\mu_{g}}{v_{f}}+\frac{a}{v_{f}}\left(\gamma_{1}+\gamma_{2}\|x\|\right) .
$$

Then,

$$
\|x\| \leq \frac{|\beta|+a \eta_{h}|\alpha|+\left(\mu_{g}+a \gamma_{1}\right)|1+\alpha|}{\left(\nu_{f}-a \gamma_{2}\right)|1+\alpha|} .
$$

Thus, all assumptions of Lemma 1 are satisfied. So, $\Pi$ has at least one fixed point which is a solution for our problem.

Now, we give an example to illustrate the obtained results.

Example 1. Consider the following problem:

$$
\left\{\begin{array}{l}
\frac{\mathrm{d}}{\mathrm{d} t}\left[8 x(t)+\frac{1-\cos (t)}{4} x(t)|x(t)|-\frac{1}{6 e^{t}} \frac{1}{1+|x(t)|}\right]=\frac{1}{5}+\frac{1}{7} x(t), \quad t \in[0, \pi], \\
x(0)+16 x(\pi)+x(\pi)|x(\pi)|=\frac{1}{48} \frac{1}{1+|x(0)|}+\frac{1}{3 e^{\pi}} \frac{1}{1+|x(\pi)|}+1 .
\end{array}\right.
$$


This problem can be written as (2), where

$$
\begin{aligned}
& f(t, x(t))=8+\frac{1-\cos (t)}{4}|x(t)|, \\
& g(t, x(t))=\frac{1}{6 e^{t}} \frac{1}{1+|x(t)|}, \\
& h(t, x(t))=\frac{1}{5}+\frac{1}{7} x(t), \quad \alpha=16 \text { and } \beta=1 .
\end{aligned}
$$

We can easily verify that

$$
\begin{aligned}
& \nu_{f}=8, \\
& \lambda_{f}=\frac{1}{4}, \\
& \mu_{g}=\frac{1}{6}, \\
& \lambda_{g}=\frac{1}{6}, \\
& \lambda_{h}=\gamma_{2}=\frac{1}{7}, \\
& k_{h}=\gamma_{1}=\frac{1}{5}, \\
& \alpha=16, \\
& \beta=1, \\
& a=\pi .
\end{aligned}
$$

We have $\nu_{f}>a \gamma_{2}$; then, from Theorem 2, this problem has a at least one solution.

Now, we know that a solution exists and we seek if it is unique. We can easily verify that by using the following:

$$
\begin{aligned}
& \pi_{1}=\frac{113}{6}+\frac{33 \pi}{5}, \\
& \pi_{2}=136, \\
& \pi_{3}=\frac{33 \pi}{7} .
\end{aligned}
$$

We take $r=1$ which satisfies condition (10). Then,

$$
\omega=\frac{203}{8704}+\frac{1419 \pi}{38080}<1, \quad \pi_{3}<\pi_{2} .
$$

According to Theorem 1, we can deduce that our problem has a unique solution.

\section{Conclusion}

In this paper, we treated a hybrid differential equation with linear and nonlinear perturbations where we showed the existence and uniqueness of the solution using Banach's fixed-point theorem, and also, we used the Leray-Schauder theorem as another way to guarantee the existence of the solution, which it was used for the first time, to our knowledge, for this type of problem. In the future research, we hope to generalize this problem either at the level of the order of the derivative or the initial condition by taking inspiration from $[8,9]$.

\section{Data Availability}

The data used to support the findings of this study are available from the corresponding author upon request.

\section{Conflicts of Interest}

The authors declare that they have no conflicts of interest.

\section{Authors' Contributions}

All authors contributed equally to the writing of this paper and read and approved the final manuscript.

\section{References}

[1] B. C. Dhage, "Nonlinear quadratic first order functional integrodifferential equations with periodic boundary conditions," $D y$ namic Systems and Applications, vol. 18, pp. 303-322, 2009.

[2] B. C. Dhage and V. Lakshmikantham, "Basic results on hybrid differential equations," Nonlinear Analysis: Hybrid Systems, vol. 4, no. 3, pp. 414-424, 2010.

[3] B. C. Dhage, S. N. Salunkhe, R. P. Agarwal, and W. Zhang, "A functional differential equation in Banach algebras," Mathematical Inequalities \& Applications, vol. 8, no. 1, pp. 89-99, 2005.

[4] K. Hilal and A. Kajouni, "Boundary value problems for hybrid differential equations with fractional order," Advances in Difference Equations, vol. 2015, no. 1, pp. 2224-5804, 2015.

[5] Y. Zhou, F. Jiao, and J. Li, "Existence and uniqueness for fractional neutral differential equations with infinite delay," Nonlinear Analysis: Theory, Methods \& Applications, vol. 71, no. 7-8, pp. 3249-3256, 2009.

[6] B. C. Dhage and N. S. Jadhav, "Basic results in the theory of hybrid differential equations with linear perturbations of second type," Tamkang Journal of Mathematics, vol. 44, no. 2, pp. 171-186, 2013.

[7] A. Granas and J. Dugundji, Fixed Point Heory, Springer, New York, NY, USA, 2003.

[8] M. Muslim, A. Kumar, and R. Sakthivel, "Exact and trajectory controllability of second-order evolution systems with impulses and deviated arguments," Mathematical Methods in the Applied Sciences, vol. 41, no. 11, pp. 4259-4272, 2018.

[9] M. Malik and A. Kumar, "Existence and controllability results to second order neutral differential equation with non-instantaneous impulses," Journal of Control and Decision, vol. 7, no. 3, pp. 286-308, 2020. 